\title{
Prophage-Encoded Peroxidase in 'Candidatus Liberibacter asiaticus' Is a Secreted Effector That Suppresses Plant Defenses
}

\author{
Mukesh Jain, Laura A. Fleites, and Dean W. Gabriel \\ Department of Plant Pathology, University of Florida, Gainesville, FL 32611-0680, U.S.A. \\ Submitted 2 July 2015. Accepted 15 August 2015.
}

\begin{abstract}
'Candidatus Liberibacter asiaticus' is transmitted by psyllids and causes huanglongbing (HLB), a lethal disease of citrus. Most pathogenic ' $\mathrm{Ca}$. L. asiaticus' strains carry two nearly identical prophages similar to SC1 and SC2 in strain UF506. SC2 was observed to replicate as a moderately high-copy excision plasmid encoding a reactive oxygen species-scavenging peroxidase (SC2_gp095), a predicted lysogenic conversion factor. SC2_gp095 was expressed at significantly higher levels in periwinkle than in citrus and was suppressed in psyllids. SC2_gp095 was cloned in a shuttle vector and transformed into Escherichia coli and Liberibacter crescens, a culturable proxy for ' $C a$. L. asiaticus'. Transformed $L$. crescens cells showed 20 to $25 \%$ enhanced resistance to $\mathrm{H}_{2} \mathrm{O}_{2}$ on agar plates, $47 \%$ greater enzymatic activity, and enhanced growth in liquid cultures. A nonclassical secretion potential was predicted for SC2_gp095 and secretion from $L$. crescens was confirmed by enzymatic and Western blot analyses. Transient expression of SC2_gp095 in planta resulted in strong transcriptional downregulation of $\operatorname{RbohB}$, the key gatekeeper of the $\mathrm{H}_{2} \mathrm{O}_{2}$-mediated defense signaling in plants, helping explain the surprisingly long incubation period (years) before HLB symptoms appear in ' $\mathrm{Ca}$. L. asiaticus'-infected citrus. ' $\mathrm{Ca}$. L. asiaticus' peroxidase is likely a secreted, horizontally acquired effector that suppresses host symptom development, a tactic used by most biotrophic plant pathogens.
\end{abstract}

Huanglongbing (HLB, or citrus greening) has emerged as the most devastating citrus disease worldwide and is currently causing severe tree and yield losses in Florida. HLB is caused by fastidious, phloem-restricted $\alpha$-proteobacteria of three known (Candidatus) species: ' $C a$. Liberibacter asiaticus', ' $C a$. L. americanus', and ' $C a$. L. africanus'. Liberibacter is vectored and transmitted among citrus and some Rutaceae species by the Asian citrus psyllid Diaphorina citri Kuwayama (Sternorrhyncha: Psyllidae) (Gottwald 2010). 'Ca. L. asiaticus' colonization in the psyllid host is systemic, spreading inter- and intracellularly across multiple organs, including the alimentary canal and salivary glands, without, however, manifestation of any pathogenic effects (Ammar et al. 2011). 'Ca. L. asiaticus' colonization in citrus, by contrast, is limited to phloem and, possibly, companion cells (Fu et al. 2015), and the incubation period before HLB symptoms appear is surprisingly long ( 1 to 3 years after the initial infections [Chiyaka et al. 2012]).

Corresponding author: D. W. Gabriel; E-mail: dgabr@ufl.edu

(c) 2015 The American Phytopathological Society
Most pathogenic ' $\mathrm{Ca}$. L. asiaticus' "strains" (' $\mathrm{Ca}$. L. asiaticus' has not been cultured) examined to date carry two similar prophages that are nearly identical and syntenic to SC1 and SC2 (GenBank accession numbers NC_019549 and NC_019550, respectively) of strain UF506 (Zhang et al. 2011). SC1 becomes replicative in planta, lytic genes are expressed, and bacteriophage particles are readily formed in the artificial host periwinkle (Catharanthus roseus) (Fleites et al. 2014; Zhang et al. 2011); phage particle formation has also been recently observed with a ' $\mathrm{Ca}$. L. asiaticus' strain in citrus (Fu et al. 2015). By contrast, $\mathrm{SC} 2$ is a replicative excision plasmid lacking lytic genes. Based on the genome sequence of ' $\mathrm{Ca}$. L. asiaticus' Psy62, which originally was thought to be missing an SC2 equivalent, reductive evolution had evidently stripped ' $\mathrm{Ca}$. L. asiaticus' of all genes needed for defense against reactive oxygen species (ROS) (Duan et al. 2009). However, the discovery of SC2 revealed the potential of SC2 for lysogenic conversion. Both a putative peroxidase (SC2_gp095) and glutathione peroxidase (SC2_gp100) are encoded on SC2 and these were suggested to provide one or more critical ROS defenses (Zhang et al. 2011).

ROS include hydrogen peroxide $\left(\mathrm{H}_{2} \mathrm{O}_{2}\right)$, superoxide anions $\left(\mathrm{O}_{2}^{-}\right)$, hydroxyl radicals $\left(\mathrm{OH}^{-}\right)$, and the ephemeral singlet oxygen $\left({ }^{1} \mathrm{O}_{2}\right)$ and are natural by-products of cellular metabolism. Stringent spatiotemporal regulation of ROS generation and detoxification pathways is essential to prevent oxidation of cellular macromolecules (Halliwell 2006). Peroxidases (EC 1.11.1.x) represent an expanding and heterogeneous group of distinct oxidoreductase enzymes implicated in detoxification of ROS and diverse biological functions, such as signal transduction, lignin biosynthesis, seed germination, senescence, and host-pathogen interactions (Passardi et al. 2005). Peroxidases differ from catalases (EC 1.11.1.6), as the latter disproportionates $\mathrm{H}_{2} \mathrm{O}_{2}$ to oxygen and water, whereas peroxidases can typically use a wide range of electron donors, including glutathione, thioredoxins, nicotinamide adenine dinucleotide phosphate (NADPH), cytochrome c, auxins, phenolic compounds, lignin precursors, secondary metabolites, lipid peroxides, and organic dyes at low physiological levels of $\mathrm{H}_{2} \mathrm{O}_{2}$ (Mishra and Imlay 2012).

Plants detect pathogen invasion in multiple ways, initially involving recognition of pathogen-associated molecular patterns (PAMPs), including direct detection of molecules of microbial origin, such as lipopolysaccharides and flagellin, and detection of cell damage caused by microbial enzymes (Boller and Felix 2009; Jones and Dangl 2006; Segonzac and Zipfel 2011). An apoplastic oxidative burst in plant cells in response to PAMPs is mediated through the action of NADPH oxidases (or respiratory burst oxidase homologs [RBOH]) (Daudi et al. 2012). 
Recent advances in Arabidopsis demonstrate that pathogen recognition and downstream signaling outputs are achieved via binding of PAMPs, such as the bacterial flagellin epitope flg22 to the membrane receptor complex FLS2 (FLAGELLINSENSING 2) that prompts a transient cytosolic $\mathrm{Ca}^{2+}$ influx, activation of CDPK $\left(\mathrm{Ca}^{2+}\right.$-dependent protein kinase) cascades, and $\mathrm{RBOH}$-mediated ROS production. Additionally, a parallel phosphorylation event releases the cytoplasmic receptor-like kinase BIK1 (BOTRYTIS-INDUCED KINASE 1), which is a direct substrate of the FLS2 complex. BIK1 positively regulates the FLS2-triggered $\mathrm{Ca}^{2+}$ influx and also phosphorylates $\mathrm{RBOHD}$, leading to a rapid $\mathrm{Ca}^{2+}$-independent oxidative burst (Li et al. 2014).

Plant RBOH proteins (10 isoforms in Arabidopsis) are a family of highly conserved intrinsic plasma membrane proteins that catalyze the formation of the superoxide anion by transferring an electron from intracellular NADPH to an apoplastic molecule of oxygen. Extremely reactive and short-lived superoxide radicals are readily converted to membrane permeable $\mathrm{H}_{2} \mathrm{O}_{2}$, either spontaneously or enzymatically, by superoxide dismutase (SOD) (Miller et al. 2009). Multilayered regulation of $\mathrm{RBOH}$-mediated generation of $\mathrm{H}_{2} \mathrm{O}_{2}$ is a prerequisite for the initiation and continuity of a rapid, selfpropagating, long-distance, systemic ROS wave imperative to induction of systemic acquired resistance and innate plant immunity or basal resistance (Baxter et al. 2014; Kadota et al. 2015; Miller et al. 2009).

Incompatible host-pathogen interaction events are marked by $\mathrm{H}_{2} \mathrm{O}_{2}$ accumulation in a biphasic manner, the second phase of the oxidative burst being responsible for the establishment of effective disease resistance (Grant and Loake 2000). During compatible interactions, only the first peak of low-magnitude nonspecific apoplastic ROS burst is observed. A substantial body of work with biotrophic and hemibiotrophic plant pathogens has established that successful infections are contingent upon i) detoxifying the first wave of ROS (primarily $\mathrm{H}_{2} \mathrm{O}_{2}$ ) and ii) suppressing basal plant defenses, including ROS elicitation and resultant mitogen-activated protein kinase cascades, expression of pathogenesis-related (PR) genes and callose deposition (Kadota et al. 2015).

Despite the availability of complete genomic DNA sequences of several ' $\mathrm{Ca}$. L. asiaticus' strains (Duan et al. 2009; Katoh et al. 2014; Lin et al. 2013) and 'Ca. L. americanus' (Wulff et al. 2014), genes governing ' $\mathrm{Ca}$. L. asiaticus' host range and the ability to colonize either psyllid or citrus or to cause symptoms in citrus are largely unknown. Furthermore, all attempts to culture ' $\mathrm{Ca}$. L. asiaticus', ' $\mathrm{Ca}$. L. americanus', or ' $C a$. L. africanus' in axenic media have so far remained unsuccessful, making functional genomics difficult. Only a single strain from a single species of Liberibacter, L. crescens BT-1, originally isolated from the sap of mountain papaya (Carica stipulata $\times$ C. pubescens), has been cultured in vitro (Leonard et al. 2012). L. crescens has no known insect host and attempts at reinoculation of $L$. crescens into many plants have remained futile, warranting speculation that $L$. crescens may no longer be parasitic or pathogenic. However, L. crescens has proved useful as a genetically tractable proxy for ' $\mathrm{Ca}$. L. asiaticus' functional genomics (Fleites et al. 2014). In the present study, SC2_ gp095 alone and in tandem with SC2_gp100 were cloned in a wide host range (repW) shuttle vector under control of the lac $Z$ promoter and were transformed into L. crescens. The objectives were to i) functionally validate SC2_gp095 as a peroxidase in ' $\mathrm{Ca}$. L. asiaticus', ii) confirm extracellular secretion of the SC2_gp095 peroxidase, and iii) examine its potential to suppress a key innate plant immune reaction ( $\mathrm{RBOH}$-mediated $\mathrm{H}_{2} \mathrm{O}_{2}$ signaling) and its role in lysogenic conversion.

\section{RESULTS}

SC2_gp095 (putative peroxidase) and SC2_gp100 (putative glutathione peroxidase)

are upregulated in planta relative to the insect host.

To assess the levels of expression of putative peroxidase genes encoded by ' $C a$. L. asiaticus' bacteriophage SC2 in different infected hosts, reverse transcription quantitative polymerase chain reaction (RT-qPCR) was carried out in ' $\mathrm{Ca}$. L. asiaticus'-infected citrus, periwinkle, and psyllids. Comparative expression analyses using ' $\mathrm{Ca}$. L. asiaticus'-infected psyllids as a baseline calibrator revealed strong and significant upregulation of both SC2_gp095 and SC2_gp100 in citrus $(P=0.00043$ and 0.003848 , respectively) and periwinkle $(P=0.0000$ and 0.000021 , respectively). In psyllids, the levels of expression of both genes were negligible. The difference in expression levels of chromosomal gene prfA (used as a control) in the three hosts was not statistically significant (Fig. 1).

\section{'Ca. L. asiaticus' SC2_gp095}

encodes a functional peroxidase.

When exposed to $\mathrm{H}_{2} \mathrm{O}_{2}$, using an in vitro agar diffusion assay, both BT-1/pLF080 (lacZ::SC2_gp095) as well as BT-1/pLF082 (lacZ::SC2_gp100::SC2_gp095) cells plated as lawns displayed a significantly smaller zone of growth inhibition (25 and $19.6 \%$, respectively) around $\mathrm{H}_{2} \mathrm{O}_{2}$-soaked filter paper discs as compared with the control BT-1/pUFR071 (empty vector) cells. This indicated that enhanced peroxide degradation activity was conferred to $L$. crescens cells grown for 12 days, by both plasmids carrying SC2_gp095 either alone or in tandem with SC2_gp100 (Fig. 2). Enhanced peroxide degradation appeared attributable only to SC2_gp095, present on both plasmids. In repeated experiments, pLF082, expressing the transcriptional fusion lacZ: SC2_gp100::SC2_gp095 from a single lacZ promoter, did not further decrease the zone of growth inhibition relative to pLF080, suggesting that SC2_gp100 did not contribute to peroxide degradation. No enhancement of peroxide degradation was conferred to Escherichia coli cells by either plasmid after overnight or much longer incubation (Fig. 2).

Functional activity of SC2_gp095 was further corroborated by quantification of enzymatic degradation of $\mathrm{H}_{2} \mathrm{O}_{2}$ in a spectrophotometric assay. BT- 1 cells transformed with either pLF080 or pLF082 displayed $47 \%$ greater peroxidase activity in cell

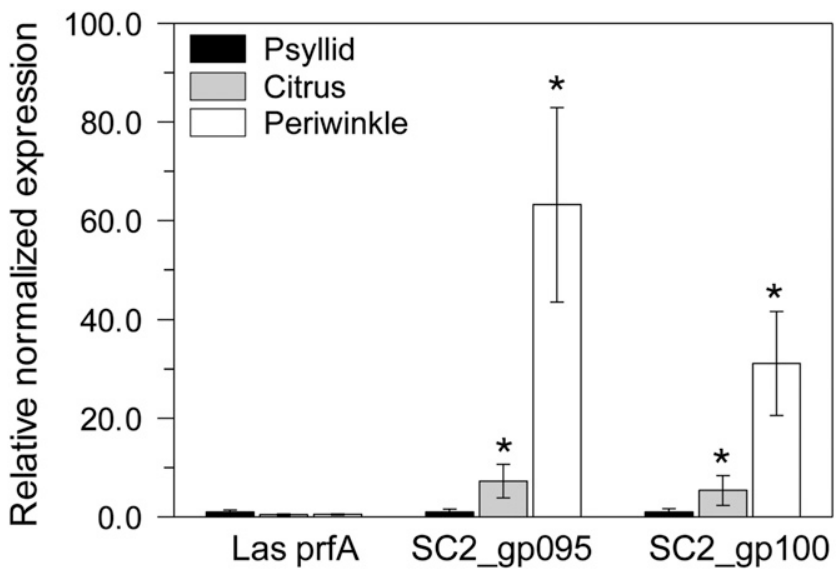

Fig. 1. Relative expression in psyllids, citrus, and periwinkle of three 'Candidatus Liberibacter asiaticus' (Las) genes: SC2 prophage-encoded peroxidase (SC2_gp095), glutathione peroxidase (SC2_gp100), and prfA. Relative transcript abundance of each gene was normalized against its expression in ' $\mathrm{Ca}$. L. asiaticus'-infected citrus. Bars represent average \pm standard error of the means. Asterisks represent significant differences in the transcript abundance between different hosts. 
lysates (4.54 and $4.53 \mathrm{mU}$, respectively) as compared with empty vector controls $(3.0 \mathrm{mU})$ assayed using $0.5-\mathrm{ml}$ cell cultures at an absorbance measured at $600 \mathrm{~nm}\left(\mathrm{Abs}_{600}\right)=0.65$ (Fig. 3A). Notably, significantly greater peroxidase activity was also observed in 50- $\mu$ l culture supernatants of BT-1 cells transformed with either pLF080 or pLF082 (1.42 and $1.36 \mathrm{mU}$, respectively), as compared with the empty vector control (0.45 mU) (Fig. 3B). However, neither of the two SC2 genes conferred an increase in the extractable peroxidase activity in similarly transformed $E$. coli cells.

\section{'Ca. L. asiaticus' SC2_gp095 peroxidase}

is secreted from $L$. crescens.

Neither of the SC2_gp095- nor SC2_gp100-encoded protein products were predicted to carry a signal peptide for extracellular bacterial secretion. However, nonclassical secretion was predicted for the SC2_gp095 protein (SecP score of 0.929821; cut-off 0.5) but not for the SC2_gp100 predicted protein (SecP score of 0.173658; cut-off 0.5) (SecretomeP 2.0) (Bendtsen et al. 2005). To determine whether the SC2_gp095 protein was secreted from $L$. crescens cells into the culture medium, Western blot analyses were carried out to detect the presence of $6 \times$ Histagged SC2_gp095 expressed from pLF080 and 6× His-tagged SC2_gp100 (together with native SC2_gp095) expressed from pLF082 in transformed $L$. crescens and E. coli cell lysates and their respective culture supernatants (Fig. 4). Monoclonal Histag antibodies specifically revealed an approximately $28-\mathrm{kDa}$

A
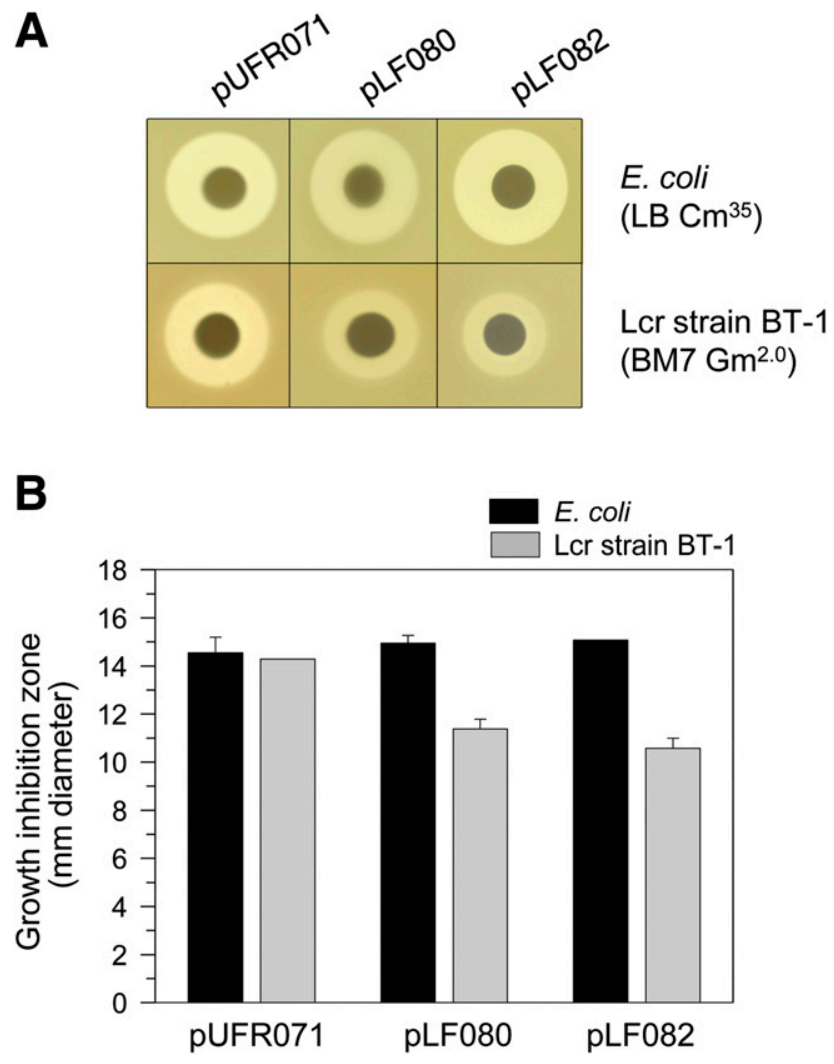

Fig. 2. Expression of peroxidase (SC2_gp095) in Liberibacter crescens (Lcr) enhances in vitro resistance to $\mathrm{H}_{2} \mathrm{O}_{2}$. A, Agar diffusion assay showing filter discs soaked in $3 \% \mathrm{H}_{2} \mathrm{O}_{2}$ and surrounding cleared zone on lawns of Escherichia coli or L. crescens cells carrying pUFR071 (empty vector control), pLF080 (lacZ::SC2_gp095), and pLF082 (lacZ::SC2_100::SC2_095), as indicated. B, Measured diameter of the cleared zones as depicted in A. The data were scored after overnight incubation for E. coli and after 12 days for $L$. crescens and represent average \pm standard error for three independent experiments. protein (SC2_gp095) in both E. coli/pLF080 and BT-1/pLF080 cell lysates. The presence of the 28-kDa SC2_gp095 His-tagged protein was only observed in the BT-1/pLF080 culture supernatant, indicating that the SC2_gp095-encoded peroxidase was secreted from intact $L$. crescens cells into the culture medium. Likewise, an approximately 10-kDa protein (SC2_gp100) was

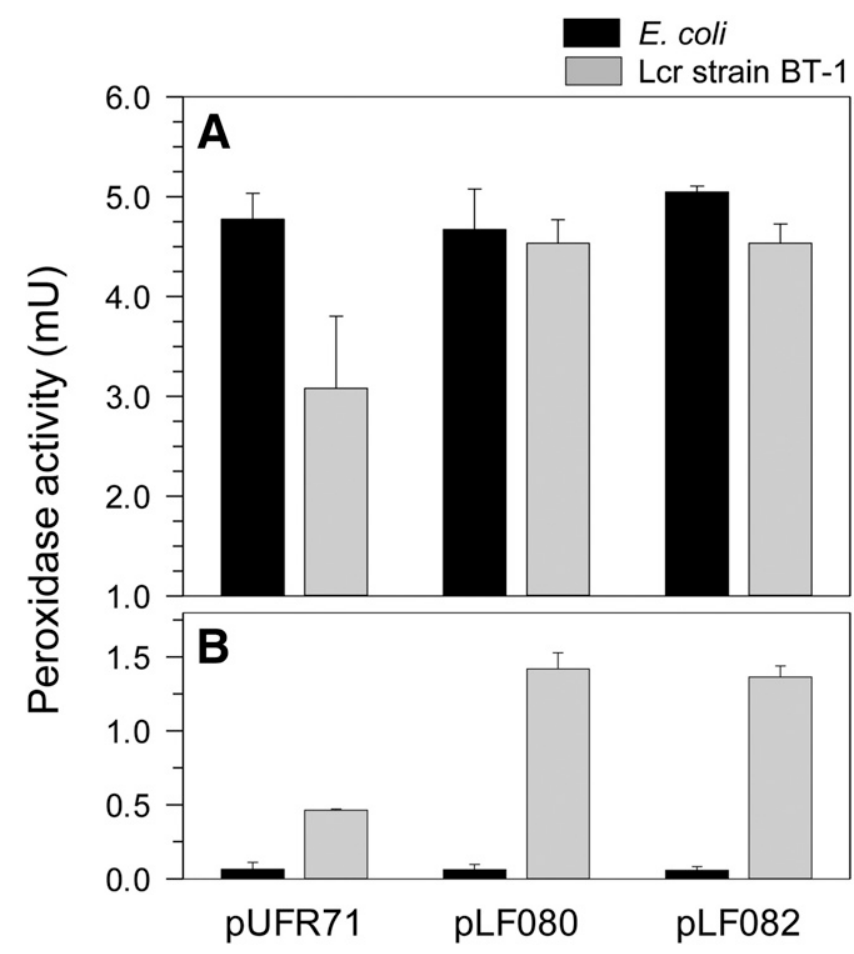

Fig. 3. Quantification of peroxidase activity in Escherichia coli and Liberibacter crescens BT-1 cells carrying pUFR071 (empty vector control), pLF080 (lacZ::SC2_gp095), and pLF082 (lacZ::SC2_100::SC2_095) and in respective culture supernatants. The cultures were grown either overnight (E. coli) or for 5 days (Liberibacter crescens) and were adjusted to an absorbance measured at $600 \mathrm{~nm}\left(\mathrm{Abs}_{600}\right)=0.65$. The enzyme activity was measured in $\mathbf{A}, 0.5 \mathrm{ml}$ of cell lysate or $\mathbf{B}, 50 \mu \mathrm{l}$ of culture supernatant. The data represent average \pm standard error for three independent experiments.

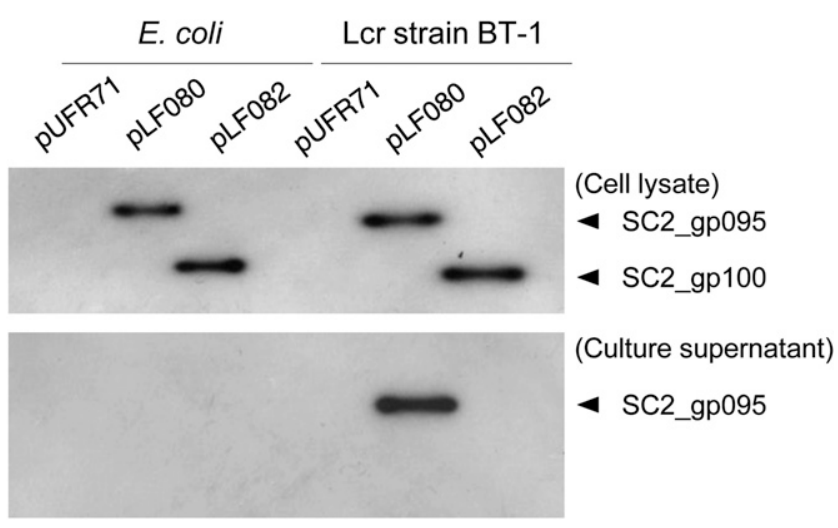

Fig. 4. SC2 prophage-encoded peroxidase (SC2_gp095) is an extracellular enzyme in Liberibacter crescens BT-1. Using $15 \mu \mathrm{g}$ of total soluble protein extracts from Escherichia coli and L. crescens BT-1 cells carrying pUFR071 (empty vector control), pLF080 (lacZ::SC2_gp095), and pLF082 (lacZ::SC2_100::SC2_095) were resolved on an 8 to $16 \%$ sodium dodecyl sulfate-polyacrylamide electrophoresis gel. The Western blot was developed using anti-6×His epitope tag primary monoclonal antibody (1:2,000 dilution) and antimouse alkaline phosphatase immunoglobulin $\mathrm{G}$ conjugate. 
detected in pLF082-transformed E. coli and L. crescens cell lysates but not in the supernatants of either species.

Lack of the relatively smaller His-tagged SC2_gp100 (10 kDa) protein in L. crescens supernatants ruled out the possibility of artifactual protein leakage due to bacterial lysis or lack of L. crescens cellular integrity. Lack of the His-tagged SC2_gp095 protein in $E$. coli supernatants indicated that the nonclassical secretion mechanism by which the protein is secreted may be species-specific or species-limited.

'Ca. L. asiaticus' peroxidase (SC2_gp095)

provides a growth advantage to $L$. crescens in culture.

L. crescens BT-1 transformed with empty vector pUFR071 reached stationary growth phase with an optical density $\left(\mathrm{Abs}_{600}\right)$ of 0.49 in 5 days of culture in BM7 liquid medium, whereas the cultures harboring either pLF080 (SC2_gp095) or pLF082 (SC2_gp095 and SC2_gp100) attained a maximum optical density $\left(\mathrm{Abs}_{600}\right)$ of 0.67 and 0.65 , respectively (Fig. 5). This $37 \%$ increase in cell density was confirmed by cell counts, which were significantly higher for BT-1/pLF080 $\left(6.8 \times 10^{8} \mathrm{CFU} / \mathrm{ml}\right)$ and for BT-1/pLF082 $\left(6.9 \times 10^{8} \mathrm{CFU} / \mathrm{ml}\right)$ than that achieved by BT-1/ pUFR071 $\left(5.7 \times 10^{7} \mathrm{CFU} / \mathrm{ml}\right)$. As previously seen in the $\mathrm{H}_{2} \mathrm{O}_{2}$ agar diffusion assay (Fig. 2) and enzymatic characterization (Fig. 3), SC2_gp100 (in pLF082) did not provide any additional growth advantage to $L$. crescens in culture above that provided by SC2_gp095 alone (in pLF080).

\section{Transient expression}

of ' $\mathrm{Ca}$. L. asiaticus' $\mathrm{SC2} 095$ in tobacco.

SC2_gp095 peroxidase function and its significance in planta were investigated by transient expression from plasmid pMJ030, using Agrobacterium tumefaciens GV2260 in the presence and absence of $50 \mu \mathrm{M} \mathrm{H}_{2} \mathrm{O}_{2}$. In the absence of peroxide induction, no significant change in $N b R b o h B$ transcript abundance was observed between 2 and 8 days postinfiltration (dpi) of GV2260 carrying either pBI121 (GUS [ $\beta$-glucuronidase] expression vector) or SC2_gp095 (Fig. 6A). The addition of $50 \mu \mathrm{M} \mathrm{H}_{2} \mathrm{O}_{2}$ to the suspension of GV2260/pBI121 used for infiltration caused a reproducibly sharp increase in $N b R b o h B$ transcript abundance from 2 to $8 \mathrm{dpi}$ (Fig. 6B). However, transient overexpression of SC2_gp095 on pMJ030 in the presence of $50 \mu \mathrm{M} \mathrm{H} \mathrm{H}_{2} \mathrm{O}_{2}$

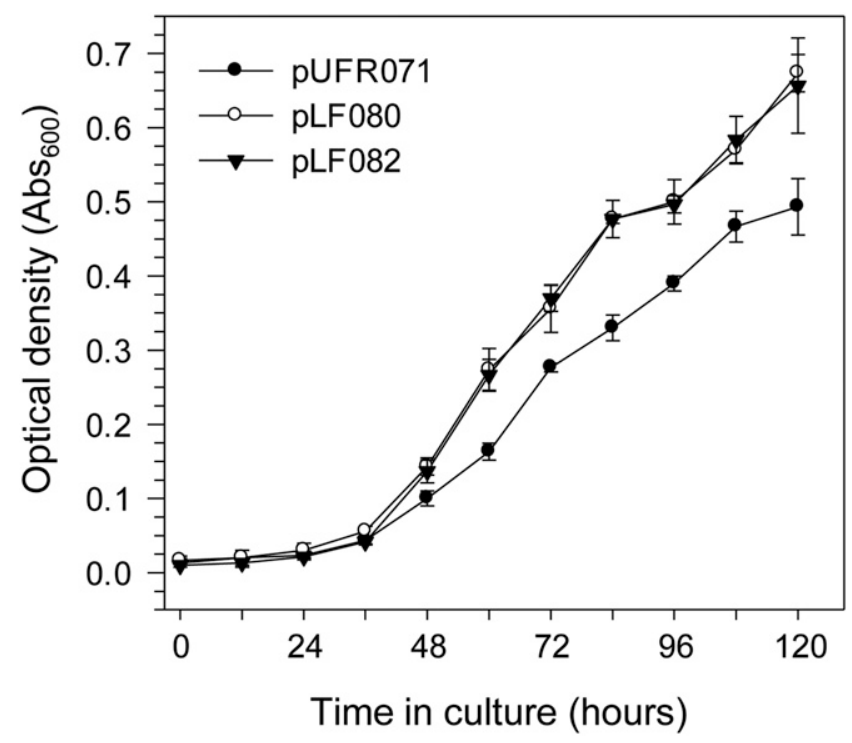

Fig. 5. Growth kinetics of Liberibacter crescens BT-1 carrying pUFR071 (empty vector control), pLF080 (lacZ::SC2_gp095), and pLF082 (lacZ:: SC2_100::SC2_095) in BM7 medium. efficiently and significantly alleviated the sharp rise in $N b R b o h B$ transcriptional activity $(P=0.000131,0.000123$, and 0.000311 , respectively) at 4,6 , and $8 \mathrm{dpi}$ (Fig. 6B), thus providing clear evidence that $\mathrm{SC} 2$ _gp095 detoxified $\mathrm{H}_{2} \mathrm{O}_{2}$ in planta and thereby suppressed transcriptional activation of $\mathrm{RbohB}$.

\section{DISCUSSION}

Several pathogenic bacteria harbor prophages encoding lysogenic conversion genes that are proven or suspected virulence factors. Although nonessential for the phage life cycle, lysogenic conversion genes impact the phenotype or fitness of the lysogen via elaborating extracellular toxins, antigenic determinants, host effector proteins, enzymes that aid in intracellular survival, and proteins facilitating bacterial attachment to host cells (Brüssow et al. 2004). The genomes of all three sequenced pathogenic Liberibacters, ' $\mathrm{Ca}$. L. asiaticus' (Zhang et al. 2011), 'Ca. L. solanacearum' (Lin et al. 2011), and ' $\mathrm{Ca}$. L. americanus' (Wulff et al. 2014) each contain two prophages encoding genes suspected of lysogenic conversion. The discernible increase in phage copy number and visual observation of phage particles in ' $\mathrm{Ca}$. L. asiaticus'-infected periwinkle (Zhang et al. 2011) and citrus (Fu et al. 2015) suggests that the SC1 lytic cycle is inducible, and phage induction may provide a tangible tool for the therapeutic control of HLB in citrus.

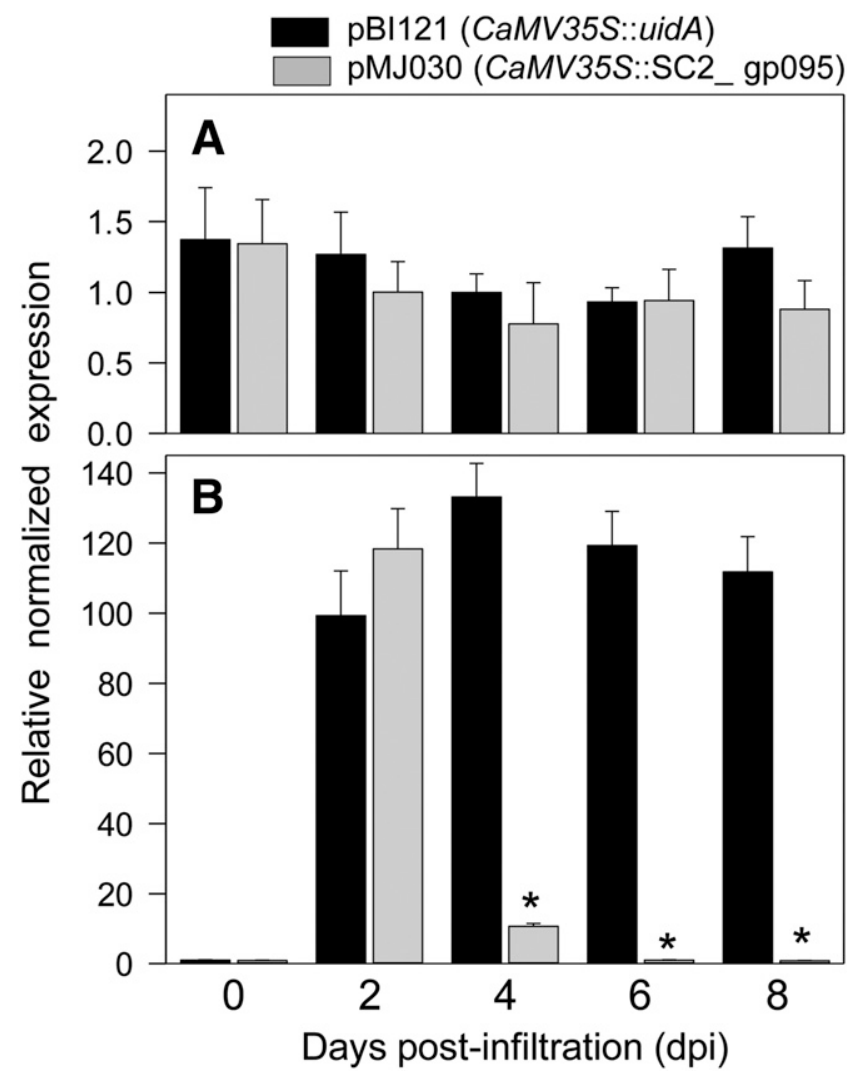

Fig. 6. Transient expression of SC2_gp095 alleviates $\mathrm{H}_{2} \mathrm{O}_{2}$-induced transcriptional upregulation of defense-related gene $\mathrm{NbRbohB}$ in planta. Nicotiana benthamiana leaves were infiltrated with Agrobacterium tumefaciens GV2260 carrying the binary vector pBI121 (CaMV35S::uidA) or pMJ030 (CaMV35S::SC2_gp095) A, without $\mathrm{H}_{2} \mathrm{O}_{2}$ and $\mathbf{B}$, along with $50 \mu \mathrm{M} \mathrm{H}_{2} \mathrm{O}_{2}$. Infiltrated tissues from three individual leaves were excised at $0,2,4,6$, and 8 days postinfiltration (dpi) and were used for RNA extraction and quantitative reverse transcription-polymerase chain reaction analyses. The $N b R b o h B$ expression data were normalized against elongation factor $1 \alpha(N b E F 1 \alpha)$. Asterisks represent significant differences between the treatment means. 
Genome sequencing of many proteobacteria typically reveals the presence of multiple mono- and bifunctional catalases, catalase/peroxidases, nonheme (Mn)-catalases, and thioredoxin peroxidases within a bacterial species that are essential for ROS detoxification and maintenance of redox poise (Klotz and Loewen 2003). However, maintenance of redox poise in Liberibacter species is limited to four enzymes, i.e., a single SOD, thioredoxin, peroxiredoxin, and glutathione oxidoreductase (Duan et al. 2009; Lin et al. 2011; Wulff et al. 2014); none of these enzymes could provide significant defense against exogenous ROS. We hypothesized that the SC2 prophageencoded peroxidase might provide an essential (and the only) defense against ROS generated by the infected plant cell. The functional data presented here represent the first report identifying a ' $\mathrm{Ca}$. L. asiaticus' virulence factor essential for initial colonization and survival in its plant host.

The RBOH-mediated oxidative burst and downstream signaling cascades are essential components of plant defense against invading pathogens (Kadota et al. 2015). In Arabidopsis, RBOHD-generated peroxide participates in long distance cell-to-cell self-propagating signaling in response to pathogen attack (or abiotic stress), and its propagation is severely attenuated upon suppression of $\mathrm{H}_{2} \mathrm{O}_{2}$ accumulation along the systemic path (Miller et al. 2009). Notably, phloem sap and sieve tube elements have been shown to serve as checkpoints for generation, amplification, and propagation as well as transport conduits for defense-related signals such as $\mathrm{H}_{2} \mathrm{O}_{2}$ (Van Bel and Gaupels 2004). Given that the ' $\mathrm{Ca}$. L. asiaticus' SC2_gp095 encodes an extracellular and functionally active $\mathrm{H}_{2} \mathrm{O}_{2}$ scavenging enzyme both in vitro (Figs. 2, 3, and 4) and in planta (Fig. 6), it is, therefore, highly likely that ' $\mathrm{Ca}$. L. asiaticus' evades early detection in phloem by virtue of its secreted peroxidase activity and, eventually, builds up a significant titer in citrus over several years before manifesting any disease symptoms, resulting in the exceedingly long incubation period often associated with HLB progression.

Several lines of evidence lend support to the argument that suppression of $\mathrm{H}_{2} \mathrm{O}_{2}$ generation and signaling events during early stages of ' $\mathrm{Ca}$. L. asiaticus' and ' $\mathrm{Ca}$. L. americanus' infection play a crucial role in subsequent colonization of its plant host. First, downregulation of NADPH oxidase/RBOHD transcriptional activity in ' $C a$. L. asiaticus'- and ' $C a$. L. americanus'-infected citrus leaves was observed by Mafra et al. (2013). Second, proteomic profiling of ' $\mathrm{Ca}$. L. asiaticus'infected grapefruit leaves revealed decreased accumulation of several $\mathrm{H}_{2} \mathrm{O}_{2}$-scavenging as well as responsive enzymatic functions, including catalase, ascorbate peroxidase, thioredoxin, isoflavone reductase, cinnamoyl-CoA-reductase, and caffeoyl $\mathrm{CoA} O$-methyltransferase, despite a discernible upregulation of an entire repertoire of (a)biotic stress-inducible PR proteins in the symptomatic leaves (Nwugo et al. 2013). Third, Slisz and coworkers (2012) observed a significant decline in proline concentration in ' $\mathrm{Ca}$. L. asiaticus'-infected citrus fruits and suggested that suppression of the defense-related $\mathrm{H}_{2} \mathrm{O}_{2}$ burst was the cause. In plants, stress-inducible accumulation of proline is dependent upon $\mathrm{H}_{2} \mathrm{O}_{2}$-mediated sequential activation of the glutamate and ornithine pathways of proline biosynthesis and the simultaneous inhibition of proline degradation (Yang et al. 2009).

In addition to suppression of $\mathrm{H}_{2} \mathrm{O}_{2}$ generation and signaling events during early stages of ' $\mathrm{Ca}$. L. asiaticus' and ' $\mathrm{Ca}$. L. americanus' infection, ' $\mathrm{Ca}$. L. asiaticus' peroxidase may also be important for scavenging host-generated $\mathrm{H}_{2} \mathrm{O}_{2}$. Scavenging of host-generated $\mathrm{H}_{2} \mathrm{O}_{2}$ has been shown to be essential for successful virulence and pathogenicity of several plant pathogens, including Ustilago maydis in maize (Molina and Kahmann 2007), Alternaria alternata and Xanthomonas citri in citrus (Lin et al. 2009; Tondo et al. 2010), and X. campestris pv. campestris in Chinese radish (Jittawuttipoka et al. 2009). Host-generated $\mathrm{H}_{2} \mathrm{O}_{2}$ can readily permeate through bacterial cell walls and oxidize $\mathrm{Fe}^{2+}$ via Fenton chemistry. Subsequent displacement of $\mathrm{Fe}^{3+}$ from the active sites of Fe-dependent mononuclear enzymes and mismetallation by $\mathrm{Zn}$ compromises enzymatic activity and imposes metabolic bottlenecks, thus halting bacterial growth and cell division (Imlay 2014). Similarly, ROS produced internally during bacterial respiration is also detrimental to cell viability absent an efficient scavenging mechanism (Cabiscol et al. 2000). The observed growth advantage conferred to $L$. crescens by SC2_gp095 (Fig. 5) likely involves direct $\mathrm{H}_{2} \mathrm{O}_{2}$ detoxification effects, both from the secreted as well as the retained cytoplasmic pool of peroxidase.

Both ' $C a$. L. asiaticus' and ' $C a$. L. americanus' are strongly biotrophic, but the infection strategies appear divergent. ' $\mathrm{Ca}$. L. asiaticus' causes even less severe symptoms than ' $\mathrm{Ca}$. L. americanus' despite growing to higher titers in citrus than ' $\mathrm{Ca}$. L. americanus' (Lopes et al. 2009). The peroxidase encoded by SC2_gp095 shows only partial homology (with at best 22\% similarity) with predicted ' $C a$. L. americanus' proteins; ' $C a$. L. americanus' may not require a peroxidase. By contrast with the ' $\mathrm{Ca}$. L. americanus' infection strategy, which appears to emphasize nearly complete loss of PAMP elicitors, including flagellin and nearly all lipopolysaccharide genes (Wulff et al. 2014), 'Ca. L. asiaticus' infection may be more reliant upon active dampening of the innate immune response and downregulation of its PAMP elicitors. Transient expression of the ' $C a$. L. asiaticus' flagellin gene $f l a_{\text {Las }}$ elicits the innate immune reaction in Nicotiana benthamiana, including increased $\mathrm{H}_{2} \mathrm{O}_{2}$ generation and callose deposition (Hao et al. 2014; Zou et al. 2012). The ' $C a$. L. asiaticus' flagellin structural domain was specifically downregulated in ' $\mathrm{Ca}$. L. asiaticus'-infected citrus leaves as compared with its insect host, despite significant upregulation of several other genes dedicated to flagellar assembly (Yan et al. 2013).

Symptoms akin to micronutrient deficiency associated with HLB are in line with the observed depletion of elemental $\mathrm{Zn}$ and $\mathrm{Fe}$ in ' $\mathrm{Ca}$. L. asiaticus'-infected citrus leaves (Masaoka et al. 2011; Nwugo et al. 2013). Consistent with these observations, ' $C a$. L. asiaticus' $z n u A B C$ encodes a functional high efficiency Zn transport system (Vahling-Armstrong et al. 2012). A considerable increase in pathogen load coupled with the associated $\mathrm{Zn}$ deficit in ' $\mathrm{Ca}$. L. asiaticus'-infected citrus cells that occurs during advanced stages of HLB should aggravate the oxidative burden in the infected cells, leading to stereotypical blotchy mottle and chlorosis in the leaves. Physiological limitation of the essential micronutrient $\mathrm{Zn}$ in the infected leaves leads to oxidative damage, primarily through diminished SOD activity and glutathione regeneration, enhanced RBOHmediated superoxide generation, and disruption of mitochondrial electron transport and photosynthetic $\mathrm{CO}_{2}$ fixation (Cakmak 2000; Eide 2011). Such molecular damage to the host cell physiology might lead to HLB symptoms, even without any specifically offensive additional pathogenicity factors.

Empirical evidence provided here confirms that the SC2 prophage-encoded peroxidase is a lysogenic conversion factor important for the fitness and survival of ' $\mathrm{Ca}$. L. asiaticus' within infected phloem cells by suppression of host defenses and scavenging ROS. Increased survival of initial ' $\mathrm{Ca}$. L. asiaticus' infections after introduction by psyllids into citrus phloem, as well as use of a strategy of avoidance of pathogenicity effectors and reduced PAMP production, helps explain the lengthy incubation period for HLB caused by ' $\mathrm{Ca}$. L. asiaticus' (a period spanning several years). Eventual symptom development is most consistent with $\mathrm{Zn}$ deficiency. We have identified, cloned, and expressed a ' $\mathrm{Ca}$. L. asiaticus' peroxidase in 
L. crescens, which is now being developed into a high throughput assay for identification of chemical inhibitors of peroxidase that could enhance native citrus resistance to ' $\mathrm{Ca}$. L. asiaticus'.

\section{MATERIALS AND METHODS}

\section{Bacterial strains and growth conditions.}

The relevant characteristics, source, or reference for the bacterial strains and plasmids used in this study are listed in Table 1. E. coli and A. tumefaciens strains were grown in Luria-Bertani (LB) medium at 37 and $28^{\circ} \mathrm{C}$, respectively. L. crescens BT- 1 was maintained on BM7 medium containing $2 \mathrm{~g}$ of $\alpha$-ketogluterate, $10 \mathrm{~g}$ of $\mathrm{N}$-(2-acetamido)-2-aminoethanesulfonic acid (ACES) buffer, and $3.75 \mathrm{~g}$ of $\mathrm{KOH}$ in $550 \mathrm{ml}$ of water, $\mathrm{pH} 6.9$, followed by the addition of filter-sterilized $300 \mathrm{ml}$ of fetal bovine serum (HyClone Laboratories, Logan, UT, U.S.A.) and $300 \mathrm{ml}$ of modified Grace's insect culture medium (TNM-FH; HyClone Laboratories) with gentle shaking at $150 \mathrm{rpm}$ at $28^{\circ} \mathrm{C}$ (Fleites et al. 2014). Antibiotics were used as needed at the following concentrations ( $\mu \mathrm{g} / \mathrm{ml})$ : ampicillin, 100; kanamycin, 50; rifampicin, 75; and gentamycin, 2.

RNA extraction and RT-qPCR from ' $\mathrm{Ca}$. L. asiaticus'infected psyllids, citrus, and periwinkle.

Psyllids maintained on ' $C a$. L. asiaticus'-infected citrus were flash-frozen and pooled samples of five psyllids per batch were ground with a mortar and pestle in lysis buffer RLT (provided with the RNeasy plant mini kit; Qiagen, Valencia, CA, U.S.A.). RNA extraction from ' $C a$. L. asiaticus'-infected psyllids, citrus, and periwinkle and subsequent RT-qPCR was performed using a CFX96 Touch real-time PCR detection system (Bio-Rad, Hercules, CA, U.S.A.), as described by Fleites et al. (2014). Two technical replicates were performed for at least six biological replicates for RNA derived from ' $\mathrm{Ca}$. L. asiaticus'-infected source material, along with healthy controls, no-template controls, and no-reverse transcription controls. Relative expression levels, which were normalized using chromosomal reference gene $g y r B$, citrus values as calibrator controls and corrected for amplification efficiency were calculated by the $\Delta \Delta \mathrm{C}_{\mathrm{t}}$ method (Livak and Schmittgen 2001). Data analyses and Student's $t$ tests $(\alpha=0.05)$ were performed using the Bio-Rad CFX Manager Software Package 3.0.

\section{Bioinformatic analyses for potential secretion signals for SC2_gp095 and SC2_gp100.}

SignalP version 4.1 (Petersen et al. 2011) was used to identify potential secretion signals from protein sequences, using both default (discrimination $[D]$ score cut-off of 0.45$)$ and sensitive $(D$ score cut-off of 0.34 ) values. Prediction of nonclassical secretion was performed using SecretomeP (Bendtsen et al. 2005).

\section{Construction of peroxidase expression vectors and transformation in $L$. crescens BT-1.}

' $C a$. L. asiaticus' DNA was extracted from the infected citrus leaves using DNeasy plant mini kit (Qiagen). The coding sequences of SC2_gp095 and SC2_gp100 were PCR-amplified using Accuprime Taq DNA polymerase high fidelity (Invitrogen, Carlsbad, CA, U.S.A.), 2x Failsafe buffer D (Epicenter, Madison, WI, U.S.A.) and primers containing a BamHI site and ShineDalgarno sequence upstream of the start codon and six histidine codons in between the penultimate codon and the stop codon. The plasmid pLF080 (containing SC2_gp095) was constructed using the primers 5' TAA GGA TCC AGG AGT AGA ACA TGG AAC AAG AGA ACA TA 3' (forward) and 5' TAT AAG CTT TCA GTG GTG GTG GTG GTG GTG TTG ATT AAG TGT TGC TTG CTT TTC 3' (reverse). The amplification product was cloned into pCR2.1TOPO (Invitrogen) and was transformed into E. coli TOP10 cells. The plasmids were verified for sequence fidelity and were digested with $B a m H I$, and the resulting

Table 1. Bacterial strains and plasmids used in this study

\begin{tabular}{|c|c|c|}
\hline Strains or plasmids & Relevant characteristics $^{\mathbf{a}}$ & Reference or source \\
\hline \multicolumn{3}{|l|}{ Strains } \\
\hline Escherichia coli TOP10 & 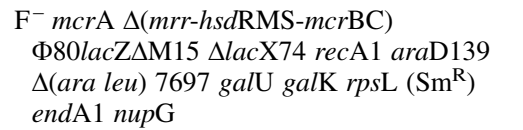 & Invitrogen \\
\hline Liberibacter crescens & $\begin{array}{l}\text { Strain BT-1, originally isolated from } \\
\text { mountain papaya }\end{array}$ & Leonard et al. 2012 \\
\hline Agrobacteriim tumefaciens GV2260 & $\begin{array}{l}\mathrm{C} 58, \mathrm{Rif}^{\mathrm{R}}, \mathrm{pGV} 2260\left(\mathrm{pTiB} 6 \mathrm{~S} 3 \Delta \mathrm{T}-\mathrm{DNA}, \mathrm{Cb}^{\mathrm{R}}\right) \text {, } \\
\text { octopine type }\end{array}$ & McBride and Summerfelt 1990 \\
\hline \multicolumn{3}{|c|}{ 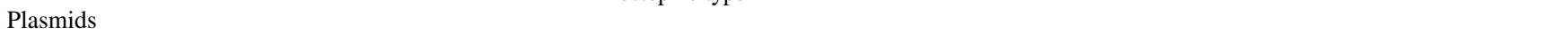 } \\
\hline pCR2.1-TOPO & $\begin{array}{l}3.9 \mathrm{~kb} \text {; polymerase chain reaction cloning } \\
\text { vector, } \mathrm{Ap}^{\mathrm{R}}, \mathrm{Kn}^{\mathrm{R}}\end{array}$ & Invitrogen \\
\hline pUFR071 & $\begin{array}{l}\text { 9.4 kb; repW, ColE1, } \mathrm{Mob}^{+}, \mathrm{lacZ}^{+}, \mathrm{Par}^{+}, \\
\mathrm{Cm}^{\mathrm{R}}, \mathrm{Gm}^{\mathrm{R}}\end{array}$ & De Feyter and Gabriel 1991 \\
\hline pBI121 & $\begin{array}{l}14.8 \mathrm{~kb} \text {; plant transformation cloning vector, } \\
\text { Bin } 19 \text { background, nptIII (bacterial } \\
\text { selection), nptII (plant selection), enhanced } \\
\text { 35S promoter, uidA reporter }\end{array}$ & Chen et al. 2003 \\
\hline pCAMBIA2301 & $\begin{array}{l}\text { 11.6 kb; pPZP family binary vector, aadA } \\
\text { (bacterial selection), nptII (plant selection), } \\
\text { enhanced 35S promoter, castor bean } \\
\text { catalase intron, uidA reporter with } \\
6 \times \text { His-tag }\end{array}$ & Hajdukiewicz et al. 1994 \\
\hline pLF080 & SC2_gp095, 6×His-tag, in pUFR071 & This study \\
\hline pLF082 & $\begin{array}{l}\text { SC2_gp100 and SC2_gp095, 6×His-tag, in } \\
\text { pUFR071 }\end{array}$ & This study \\
\hline pMJ030 & $\begin{array}{l}\text { Castor bean catalase intron from } \\
\text { pCAMBIA2301 fused in frame with } \\
\text { 'Candidatus Liberibacter asiaticus' } \\
\text { SC2_gp095, directionally cloned in pBI121 } \\
\text { to replace the C coding sequence }\end{array}$ & This study \\
\hline
\end{tabular}

\footnotetext{
${ }^{\mathrm{a}} \mathrm{Sm}^{\mathrm{R}}, \mathrm{Cb}^{\mathrm{R}}, \mathrm{Ap}^{\mathrm{R}}, \mathrm{Kn}^{\mathrm{R}}, \mathrm{Cm}^{\mathrm{R}}$, and $\mathrm{Gm}^{\mathrm{R}}=$ streptomycin, carbenicillin, ampicillin, kanamycin, chloramphenicol, and gentamycin resistance.
} 
fragment was gel-purified, followed by ligation into broad host range repW shuttle vector pUFR071 (De Feyter and Gabriel 1991). E. coli TOP10 cells were transformed with the ligation mixture and colonies were screened for orientation. Likewise, a second construct, designated pLF082 (containing SC2_gp095 and the contiguous SC2_gp100) was created using the primers 5' TAA GGA TCC AGG AGA GAG GTA TGA TTA AGGAGAT TTG AT 3' (forward) and 5' TAT AAG CTT TCA GTG GTG GTG GTG GTG GTG TTG ATT AAG TGT TGC TTG CTT TTC 3' (reverse). Transformation of L. crescens BT-1 was done by electroporation as described previously (Fleites et al. 2014).

\section{$\mathrm{H}_{2} \mathrm{O}_{2}$-resistance assay.}

To assay $\mathrm{H}_{2} \mathrm{O}_{2}$ tolerance, $L$. crescens and E. coli strains transformed with pUFR071, pLF080, or pLF082 were grown for 5 days in BM7 or overnight in selective LB media, respectively. The bacteria were harvested by centrifugation and were resuspended in the corresponding liquid medium to obtain $\mathrm{Abs}_{600}=0.65$. Resuspended bacterial solution $(1 \mathrm{ml})$ was mixed with $4 \mathrm{ml}$ of appropriate medium containing top agar $\left(7.5 \mathrm{~g} \mathrm{l}^{-1}\right.$, wt/vol $)$ and was immediately seeded on selective plates. Sterile Whatman 3MM filter paper discs $(5 \mathrm{~mm})$ were soaked with $25 \mu \mathrm{l}$ of $\mathrm{H}_{2} \mathrm{O}_{2}(3 \%$, $\mathrm{vol} / \mathrm{vol}$ ) and was placed on the plates. The zones of growth inhibition around the paper discs were measured after overnight and up to 12 days incubation for E. coli or 12 days for L. crescens.

\section{Peroxidase activity assay.}

Enzymatic peroxidase activity was measured using the Amplex red hydrogen peroxide/peroxidase assay kit (Invitrogen). Aliquots of 5-day-old L. crescens cultures $\left(0.5 \mathrm{ml}, \mathrm{Abs}_{600}=0.65\right)$ were harvested by centrifugation and were resuspended in $50 \mu \mathrm{l}$ of $0.25 \mathrm{M}$ sodium phosphate buffer ( $\mathrm{pH} 7.4)$. Likewise, $E$. coli cultures grown overnight were appropriately diluted to $\mathrm{Abs}_{600}=0.65$ and were assayed for the enzymatic activity. An equal volume of $100 \mu \mathrm{M}$ AmplexRed reagent (10-acetyl3,7-dihydroxyphenoxazine) and $2 \mathrm{mM} \mathrm{H}_{2} \mathrm{O}_{2}$ was added to the bacterial suspension and was incubated for $30 \mathrm{~min}$ in dark. The development of red-chromogenic oxidation product resorufin was measured at $560 \mathrm{~nm}$. The enzyme activity was normalized against a standard curve obtained for horseradish peroxidase. The data represent average \pm standard error for three independent experiments.

\section{Sodium dodecyl sulfate-polyacrylamide gel electrophoresis (SDS-PAGE) and protein gel blot analysis.}

Total soluble proteins from the transformed L. crescens and $E$. coli were extracted using a Qproteome bacterial protein prep kit (Qiagen). The culture supernatants were concentrated by passage through an Amicon ultra-0.5 centrifugal filter (molecular weight cut-off of $3 \mathrm{kDa}$; Millipore, Bedford, MA, U.S.A.). Protein concentration was determined using the DC protein assay kit (Bio-Rad). Precast 8 to 16\% Mini-PROTEAN TGX (Bio-Rad) SDS-PAGE gels were used for resolving $15 \mu \mathrm{g}$ of total protein and for transfer onto nitrocellulose membranes (GE Water and Process Technologies, Trevose, PA, U.S.A.) using the mini trans-blot apparatus (Bio-Rad). Anti $6 \times \mathrm{His}$ epitope tag primary monoclonal antibody (Thermo Scientific, Waltham, MA, U.S.A.) was used at a 1:2,000 dilution, and the protein gel blots were developed using the WesternBreeze chemiluminescent immunodetection kit (Invitrogen) supplied with antimouse alkaline phosphatase immunoglobulin $\mathrm{G}$ conjugate.

\section{Construction of binary vector}

for transient expression of SC2_gp095 in planta.

The castor bean catalase intron (from pCAMBIA2301) (Hajdukiewicz et al. 1994) and SC2_gp095 (from ' $\mathrm{Ca}$. L. asiaticus'- infected citrus leaf) were PCR-amplified using the following primer pairs: catalase intron, 5' TCT AGA GGA TCC CAT GGT AGA TCT GAG GGT AAA TTT CTA 3' (forward) and $5^{\prime}$ TTC TCT TGT TCA GGG ACG ACG AGT CGT CGG TTC TGT AAC TAT 3' (reverse); SC2_gp095, 5' GAC GAC TCG TCG TCC CTG AAC AAG AGA ACA TAC AAC TGA AGG AAG GAA TTG TTG ATA 3' (forward) and 5' GAG CTC CTT AAG TTA TTG ATT AAG TGT TGC TTG CTT TTC GG 3' (reverse). The PCR products were fused in frame via splice overlap PCR, utilizing the underlined sequence homology (Castañeda et al. 2005). The resulting PCR product with upstream $\mathrm{XbaI}$ and downstream $\mathrm{SacI}$ sites was cloned in E. coli TOP10 cells. Following sequence verification, the insert was

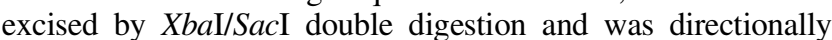
subcloned into pBI121 (Chen et al. 2003), replacing the GUS coding sequence. The resulting plasmid pMJ030 was mobilized into A. tumefacience GV2260 via electroporation.

\section{Agrobacterium-mediated transient expression of SC2_gp095 in tobacco.}

Agrobacterium-mediated transient expression assays in $N$. benthamiana were performed as described previously (Hao et al. 2014). In brief, $50 \mu \mathrm{l}$ of an overnight A. tumefaciens GV2260 broth culture was inoculated in $5 \mathrm{ml}$ of LB medium supplemented with appropriate antibiotics and $100 \mu \mathrm{M}$ acetosyringone. Bacterial cells were harvested and resuspended in induction buffer composed of $10 \mathrm{mM} \mathrm{MgCl} 2,10 \mathrm{mM}$ MES [2( $N$-morpholino)-ethanesulfonic acid] (pH 5.6) and $100 \mu \mathrm{M}$ acetosyringone. The bacterial inoculums were adjusted to optical density at $600 \mathrm{~nm}=0.6$, were incubated for $3 \mathrm{~h}$ at room temperature, and were infiltrated into the stomata of fully expanded tobacco leaves, using a 1-ml needleless blunt-end syringe. The infiltrated leaf tissues were sampled at indicated time points and were used for RNA extraction, using Trizol reagent (Invitrogen) and RT-qPCR analysis as described (Fleites et al. 2014). The expression data for $N b R b o h B$ was normalized against elongation factor $1 \alpha(N b E F 1 \alpha)$, using the previously described primer pairs (Hao et al. 2014). Student's $t$ tests $(\alpha=0.05)$ were performed using the Bio-Rad CFX Manager software package 3.0.

\section{ACKNOWLEDGMENTS}

We thank P. Rayside for excellent technical assistance and N. Killiny, Citrus Research and Education Center, University of Florida, Lake Alfred, FL, U.S.A. for providing ' $\mathrm{Ca}$. L. asiaticus'-infected psyllids. This work was supported by the Florida Citrus Research and Development Foundation (CRDF) project \#108214

\section{LITERATURE CITED}

Ammar, E.-D., Shatters, R. G., Jr., Lynch, C., and Hall, D. G. 2011. Detection and relative titer of Candidatus Liberibacter asiaticus in the salivary glands and alimentary canal of Diaphorina citri (Hemiptera: Psyllidae) vector of citrus Huanglongbing disease. Ann. Entomol. Soc. Am. 104:526-533.

Baxter, A., Mittler, R., and Suzuki, N. 2014. ROS as key players in plant stress signalling. J. Exp. Bot. 65:1229-1240.

Bendtsen, J. D., Kiemer, L., Fausbøll, A., and Brunak, S. 2005. Nonclassical protein secretion in bacteria. BMC Microbiol. 5:58.

Boller, T., and Felix, G. 2009. A renaissance of elicitors: Perception of microbe-associated molecular patterns and danger signals by patternrecognition receptors. Annu. Rev. Plant Biol. 60:379-406.

Brüssow, H., Canchaya, C., and Hardt, W.-D. 2004. Phages and the evolution of bacterial pathogens: From genomic rearrangements to lysogenic conversion. Microbiol. Mol. Biol. Rev. 68:560-602.

Cabiscol, E., Tamarit, J., and Ros, J. 2000. Oxidative stress in bacteria and protein damage by reactive oxygen species. Int. Microbiol. 3:3-8.

Cakmak, I. 2000. Tansley Review No. 111. Possible roles of zinc in protecting plant cells from damage by reactive oxygen species. New Phytol. 146:185-205. 
Castañeda, A., Reddy, J. D., El-Yacoubi, B., and Gabriel, D. W. 2005. Mutagenesis of all eight avr genes in Xanthomonas campestris pv. campestris had no detected effect on pathogenicity, but one avr gene affected race specificity. Mol. Plant-Microbe Interact. 18:1306-1317.

Chen, P. Y., Wang, C. K., Soong, S. C., and To, K. Y. 2003. Complete sequence of the binary vector pBI121 and its application in cloning T-DNA insertion from transgenic plants. Mol. Breed. 11:287-293.

Chiyaka, C., Singer, B. H., Halbert, S. E., Morris, J. G., Jr., and van Bruggen, A. H. C. 2012. Modeling huanglongbing transmission within a citrus tree. Proc. Natl. Acad. Sci. U.S.A. 109:12213-12218.

Daudi, A., Cheng, Z., O'Brien, J. A., Mammarella, N., Khan, S., Ausubel, F. M., and Bolwell, G. P. 2012. The apoplastic oxidative burst peroxidase in Arabidopsis is a major component of pattern-triggered immunity. Plant Cell 24:275-287.

De Feyter, R., and Gabriel, D. W. 1991. Use of cloned DNA methylase genes to increase the frequency of transfer of foreign genes into Xanthomonas campestris pv. malvacearum. J. Bacteriol. 173:6421-6427.

Duan, Y., Zhou, L., Hall, D. G., Li, W., Doddapaneni, H., Lin, H., Liu, L., Vahling, C. M., Gabriel, D. W., Williams, K. P., Dickerman, A., Sun, Y., and Gottwald, T. 2009. Complete genome sequence of citrus huanglongbing bacterium, 'Candidatus Liberibacter asiaticus' obtained through metagenomics. Mol. Plant-Microbe Interact. 22:1011-1020.

Eide, D. J. 2011. The oxidative stress of zinc deficiency. Metallomics 3: 1124-1129.

Fleites, L. A., Jain, M., Zhang, S., and Gabriel, D. W. 2014. 'Candidatus Liberibacter asiaticus' prophage late genes may limit host range and culturability. Appl. Environ. Microbiol. 80:6023-6030.

Fu, S., Li, Z., Tan, J., Su, H., Hartung, J., and Zhou, C. Y. 2015. Ultrastructural changes and putative phage particles observed in sweet orange leaves infected with 'Candidatus Liberibacter asiaticus'. Plant Dis. 99:320-324.

Gottwald, T. R. 2010. Current epidemiological understanding of citrus huanglongbing. Annu. Rev. Phytopathol. 48:119-139.

Grant, J. J., and Loake, G. J. 2000. Role of reactive oxygen intermediates and cognate redox signaling in disease resistance. Plant Physiol. 124:21-30.

Hajdukiewicz, P., Svab, Z., and Maliga, P. 1994. The small, versatile pPZP family of Agrobacterium binary vectors for plant transformation. Plant Mol. Biol. 25:989-994.

Halliwell, B. 2006. Reactive species and antioxidants. Redox biology is a fundamental theme of aerobic life. Plant Physiol. 141:312-322.

Hao, G., Pitino, M., Ding, F., Lin, H., Stover, E., and Duan, Y. 2014. Induction of innate immune responses by flagellin from the intracellular bacterium, 'Candidatus Liberibacter solanacearum'. BMC Plant Biol. 14:211.

Imlay, J. A. 2014. The mismetallation of enzymes during oxidative stress. J. Biol. Chem. 289:28121-28128.

Jittawuttipoka, T., Buranajitpakorn, S., Vattanaviboon, P., and Mongkolsuk, S. 2009. The catalase-peroxidase $\mathrm{KatG}$ is required for virulence of Xanthomonas campestris pv. campestris in a host plant by providing protection against low levels of $\mathrm{H}_{2} \mathrm{O}_{2}$. J. Bacteriol. 191:7372-7377.

Jones, J. D., and Dangl, J. L. 2006. The plant immune system. Nature 444: 323-329.

Kadota, Y., Shirasu, K., and Zipfel, C. 2015. Regulation of the NADPH oxidase RBOHD during plant immunity. Plant Cell Physiol. 56:1472-1480

Katoh, H., Miyata, S., Inoue, H., and Iwanami, T. 2014. Unique features of a Japanese 'Candidatus Liberibacter asiaticus' strain revealed by whole genome sequencing. PLoS One 9:e106109.

Klotz, M. G., and Loewen, P. C. 2003. The molecular evolution of catalatic hydroperoxidases: Evidence for multiple lateral transfer of genes between prokaryota and from bacteria into eukaryota. Mol. Biol. Evol. 20:1098-1112.

Leonard, M. T., Fagen, J. R., Davis-Richardson, A. G., Davis, M. J., and Triplett, E. W. 2012. Complete genome sequence of Liberibacter crescens BT-1. Stand. Genomic Sci. 7:271-283.

Li, L., Li, M., Yu, L., Zhou, Z., Liang, X., Liu, Z., Cai, G., Gao, L., Zhang, X., Wang, Y., Chen, S., and Zhou, J.-M. 2014. The FLS2-associated kinase BIK1 directly phosphorylates the NADPH oxidase RbohD to control plant immunity. Cell Host Microbe 15:329-338.

Lin, C.-H., Yang, S. L., and Chung, K.-R. 2009. The YAP1 homologmediated oxidative stress tolerance is crucial for pathogenicity of the necrotrophic fungus Alternaria alternata in citrus. Mol. Plant-Microbe Interact. 22:942-952.

Lin, H., Han, C. S., Liu, B., Lou, B., Bai, X., Deng, C., Civerolo, E. L., and Gupta, G. 2013. Complete genome sequence of a Chinese strain of 'Candidatus Liberibacter asiaticus'. Genome Announc. 1:e00184-13.

Lin, H., Lou, B., Glynn, J. M., Doddapaneni, H., Civerolo, E. L., Chen, C., Duan, Y., Zhou, L., and Vahling, C. M. 2011. The complete genome sequence of 'Candidatus Liberibacter solanacearum', the bacterium associated with potato zebra chip disease. PLoS One 6:e19135.
Livak, K. J., and Schmittgen, T. D. 2001. Analysis of relative gene expression data using real-time quantitative PCR and the $2^{-\Delta \Delta \mathrm{Ct}}$ method. Methods 25:402-408.

Lopes, S. A., Bertolini, E., Frare, G. F., Martins, E. C., Wulff, N. A., Teixeira, D. C., Fernandes, N. G., and Cambra, M. 2009. Graft transmission efficiencies and multiplication of 'Candidatus Liberibacter americanus' and ' $\mathrm{Ca}$. Liberibacter asiaticus' in citrus plants. Phytopathology 99:301-306.

Mafra, V., Martins, P. K., Francisco, C. S., Ribeiro-Alves, M., FreitasAstúa, J., and Machado, M. A. 2013. Candidatus Liberibacter americanus induces significant reprogramming of the transcriptome of the susceptible citrus genotype. BMC Genomics 14:247.

Masaoka, Y., Pustika, A., Subandiyah, S., Okada, A., Hanundin, E., Purwanto, B., Okuda, M., Okada, Y., Saito, A., Holford, P., Beattie, A., and Iwanami, T. 2011. Lower concentrations of microelements in leaves of citrus infected with 'Candidatus Liberibacter asiaticus'. Jpn. Agric. Res. Q. 45:269-275.

McBride, K. E., and Summerfelt, K. R. 1990. Improved binary vectors for Agrobacterium-mediated plant transformation. Plant Mol. Biol. 14: 269-276.

Miller, G., Schlauch, K., Tam, R., Cortes, D., Torres, M. A., Shulaev, V., Dangl, J. L., and Mittler, R. 2009. The plant NADPH oxidase RBOHD mediates rapid systemic signaling in response to diverse stimuli. Sci. Signal. 2:ra45.

Mishra, S., and Imlay, J. 2012. Why do bacteria use so many enzymes to scavenge hydrogen peroxide? Arch. Biochem. Biophys. 525:145-160.

Molina, L., and Kahmann, R. 2007. An Ustilago maydis gene involved in $\mathrm{H}_{2} \mathrm{O}_{2}$ detoxification is required for virulence. Plant Cell 19:2293-2309.

Nwugo, C. C., Duan, Y., and Lin, H. 2013. Study on citrus response to huanglongbing highlights a down-regulation of defense-related proteins in lemon plants upon ' $\mathrm{Ca}$. Liberibacter asiaticus' infection. PLoS One 8:e67442.

Passardi, F., Cosio, C., Penel, C., and Dunand, C. 2005. Peroxidases have more functions than a Swiss army knife. Plant Cell Rep. 24:255-265.

Petersen, T. N., Brunak, S., von Heijne, G., and Nielsen, H. 2011. SignalP 4.0: Discriminating signal peptides from transmembrane regions. Nat. Methods 8:785-786.

Segonzac, C., and Zipfel, C. 2011. Activation of plant pattern-recognition receptors by bacteria. Curr. Opin. Microbiol. 14:54-61.

Slisz, A. M., Breksa, A. P., 3rd, Mishchuk, D. O., McCollum, G., and Slupsky, C. M. 2012. Metabolomic analysis of citrus infection by 'Candidatus Liberibacter' reveals insight into pathogenicity. J. Proteome Res. 11:4223-4230.

Tondo, M. L., Petrocelli, S., Ottado, J., and Orellano, E. G. 2010. The monofunctional catalase KatE of Xanthomonas axonopodis pv. citri is required for full virulence in citrus plants. PLoS One 5:e10803.

Vahling-Armstrong, C. M., Zhou, H., Benyon, L., Morgan, J. K., and Duan, Y. 2012. Two plant bacteria, S. meliloti and $C a$. Liberibacter asiaticus, share functional $z n u A B C$ homologues that encode for a high affinity zinc uptake system. PLoS One 7:e37340.

Van Bel, A. J. E., and Gaupels, F. 2004. Pathogen-induced resistance and alarm signals in the phloem. Mol. Plant Pathol. 5:495-504.

Wulff, N. A., Zhang, S., Setubal, J. C., Almeida, N. F., Martins, E. C., Harakava, R., Kumar, D., Ranngel, L. T., Foissac, X., Bové, J. M., and Gabriel, D. W. 2014. The complete genome sequence of 'Cadidatus Liberibacter americanus', associated with citrus huanglongbing. Mol. Plant-Microbe Interact. 27:163-176.

Yan, Q., Sreedharan, A., Wei, S., Wang, J., Pelz-Stelinski, K., Folimonova, S., and Wang, N. 2013. Global gene expression changes in Candidatus Liberibacter asiaticus during the transmission in distinct hosts between plant and insect. Mol. Plant Pathol. 14:391-404.

Yang, S. L., Lan, S. S., and Gong, M. 2009. Hydrogen peroxide-induced proline and metabolic pathway of its accumulation in maize seedlings. J. Plant Physiol. 166:1694-1699.

Zhang, S., Flores-Cruz, Z., Zhou, L., Kang, B.-H., Fleites, L. A., Gooch, M. D., Wulff, N. A., Davis, M. J., Duan, Y.-P., and Gabriel, D. W. 2011 'Ca. Liberibacter asiaticus' carries an excision plasmid prophage and a chromosomally integrated prophage that becomes lytic in plant infections. Mol. Plant-Microbe Interact. 24:458-468.

Zou, H., Gowda, S., Zhou, L., Hajeri, S., Chen, G., and Duan, Y. 2012. The destructive citrus pathogen, 'Candidatus Liberibacter asiaticus' encodes a functional flagellin characteristic of a pathogen-associated molecular pattern. PLoS One 7:e46447.

\section{AUTHOR-RECOMMENDED INTERNET RESOURCES}

SecretomeP 2.0 server: http://www.cbs.dtu.dk/services/SecretomeP SignalP 4.1 server: http://www.cbs.dtu.dk/services/SignalP 